\title{
FREE BOUNDARY CONDITIONS FOR INTRINSIC SHELL MODELS
}

\author{
John Cagnol \\ Pôle Universitaire Léonard de Vinci, \\ Paris, France \\ John.Cagnol@devinci.fr \\ Catherine Lebiedzik* \\ University of Virginia and Pole Universitaire Léonard de Vinci, \\ Charlottesville, Virginia, USA \\ cgl5f@virginia.edu
}

\begin{abstract}
We derive the free boundary conditions and associated strong form of a shallow Kirchhoff shell model based on the intrinsic geometry methods of Michael Delfour and Jean-Paul Zolésio. Manipulations with the model result in a cleaner form where the displacement of the shell and shell boundary is written explicitly in terms of standard tangential operators.
\end{abstract}

\section{Introduction}

A wide variety of engineering and applied mathematics problems can be modeled in the context of thin shells. Many problems involve issues such as, for example, boundary feedback control, for which sophisticated mathematical analyses have been developed in the analogous plate case. The difficulty with adapting these techniques to classical shell models is one motivating factor driving the development of new modeling schemes.

The technique proposed by Michel Delfour and Jean-Paul Zolésio [6], [7], and used here, takes advantage of the intrinsic geometric properties of the shell. The model used here was introduced in [5] where the Kirchhoff hypothesis and shallowness assumption were used. We here continue our previous work on the model by deriving the free boun-

${ }^{*}$ Research supported by the National Science Foundation under Grant INT-0104431.

The original version of this chapter was revised: The copyright line was incorrect. This has been corrected. The Erratum to this chapter is available at DOI: 10.1007/978-0-387-35690-7_44

V. Barbu et al. (eds.), Analysis and Optimization of Differential Systems

(C) IFIP International Federation for Information Processing 2003 
dary conditions and associated strong from of a Kirchoff-type model presented in the language of the intrinsic geometry of Delfour and Zolésio [8], [6]. The free boundary conditions are very important from both a physical and a control point of view. These are the natural (Neumanntype) boundary conditions and correspond to the actions of bending moments and shear forces. One can apply forces and torques through these moments in order to control and stabilize the vibrating body. This has been studied extensively in the case of plates[13], [14].

Classical shell modeling is a subject well covered in many books, so here we will mention only results which are related to the work at hand. The method of integrating over thickness to derive an equation for the mid-surface of a plate or shell was pioneered by Kirchhoff [10] and Love [16]; and developed and improved by Koiter[11], [12]. The important works of Bernadou, Boisserie, Ciarlet, Miara [1], [2], [3], [4] and others analyzed Koiter's model in detail and established ellipticity of the strain energy, convergence and error estimates allowing application of conforming and non-conforming finite element methods. However, the appearance of variable coefficients in the principal part of the operator (a necessary feature of shell models) often makes theoretical analysis difficult, especially from the point of view of stabilization and control. Thus, shell models based on more geometrical arguments are currently being proposed to circumvent these problems.

The technique proposed by Michel Delfour and Jean-Paul Zolésio [6], [7], [8] (and used here) takes advantage of the intrinsic geometric properties of the shell. Here, the shell is described in terms of tangential differential operators which are defined by means of the oriented boundary distance function in $\mathbb{R}^{3}$. Sobolev spaces, Green's formula, and key inequalities such as Poincaré and Korn's inequalities are all welldefined. Delfour and Zolésio have constructed models under a variety of assumptions. The model developed in this paper was introduced in [5] where the Kirchhoff hypothesis and shallowness criterion were used.

This paper expands the shell model introduced in [5] by formulating the associated 'free' boundary conditions. Though this model was used quite successfully for an application involving uniform stability of a structural acoustic model, clearly we assumed a fully clamped shell boundary in the derivation. In contrast, in this work we assume that the shell is only clamped on a portion of the boundary, and free on the rest. The assumption that the shell is partially clamped is necessary to conclude that the elastic energy is still, in fact, coercive. In order to derive the boundary conditions we explicitly calculate the integrals over the boundary using the appropriate form of Green's formula. The 
final result is presented in terms of standard tangential operators, with coupling and lower-order terms separated in a condensed way.

\section{Intrinsic Geometric Modeling}

We here include a brief discussion of the oriented distance function and the intrinsic geometric methods of Delfour and Zolésio. The reader is referred to [8], [6] for a definitive exposition on this topic. Consider a domain $\mathcal{O} \subset \mathbb{R}^{3}$ whose nonempty boundary $\partial \mathcal{O}$ is a $C^{3}$ two-dimensional submanifold of $\mathbb{R}^{3}$. Define the oriented (or signed) distance function to $\mathcal{O}$ as

$$
b(x)=d_{\mathcal{O}}(x)-d_{\mathbb{R}^{3} \backslash \mathcal{O}}(x)
$$

where $d$ is the Euclidean distance from the point $x$ to the domain $\mathcal{O}$. In other words, $b(x)$ is simply the positive or negative distance to the boundary $\partial \mathcal{O}$, depending on if we are outside or inside the domain $\mathcal{O}$. It can be shown that for every $x \in \partial \mathcal{O}$, there exists a neighborhood where the function $\nabla b=\nu$, the unit outward external normal to $\partial \mathcal{O}$ [6]. Consider a subset $\Gamma \subseteq \partial \mathcal{O}$ which will eventually become the midsurface of our shell. We define the projection $p(x)$ of a point $x$ onto $\Gamma$ as $p(x)=x-b(x) \nabla b(x)$. Then, we define a shell $S_{h}$ of thickness $h$ as

$$
S_{h}(\Gamma) \equiv\left\{x \in \mathbb{R}^{3}: p(x) \in \Gamma,|b(x)|<h / 2\right\}
$$

Let $\Upsilon \equiv \partial \Gamma$ denote the boundary of $\Gamma$. and $(X, z)$ be the natural curvilinear coordinate system induced on the shell $S_{h}$, where the coordinate vector $X$ gives the position of a point on the mid-surface $\Gamma$, and $z \in\left(-\frac{h}{2}, \frac{h}{2}\right)$ gives the vertical (normal) distance from the midsurface. Using this notation, we also define the "flow mapping" $T_{z}(X)$ as $T_{z}(X)=X+z \nabla b(X)$ for all $X$ and $z$ in $S_{h}$. This allows us to reconstruct the action at a given height $z$ of the shell, once we know the action of the midsurface $\Gamma$. Define as $\Gamma^{z}$ the surface $T_{z}(\Gamma)$ at the 'altitude' $z$. Then, one can also describe the shell $S_{h}$ as $S_{h}=\cup_{z=-h / 2}^{h / 2} \Gamma_{z}$. The curvatures of the shell will be denoted $H$ and $K$. These can be reconstructed from the boundary distance function $b(x)$ by noting that at any point $(X, z)$, the matrix $D^{2} b$ has eigenvalues $0, \lambda_{1}, \lambda_{2}$. The curvatures are then given by $\operatorname{tr}\left(D^{2} b\right)=2 H=\lambda_{1}+\lambda_{2}$ and $K=\lambda_{1} \lambda_{2}$.

Next, we mention briefly some useful aspects of the tangential differential calculus. Given $f \in C^{1}(\Gamma)$, we define the tangential gradient $\nabla_{\Gamma}$ of the scalar function $f$ by means of the projection as

$$
\left.\nabla_{\Gamma} f \equiv \nabla(f \circ p)(x)\right|_{\Gamma}
$$

This notion of the tangential gradient is equivalent to the classical definition using an extension $F$ of $f$ in the neighborhood of $\Gamma$, i.e. 
$\nabla_{\Gamma} f=\left.\nabla F\right|_{\Gamma}-\frac{\partial F}{\partial \nu} \nu[6]$. Following the same idea we can define the tangential Jacobian matrix of a vector function $v \in C^{1}(\Gamma)^{3}$ as

$$
\left.D_{\Gamma} v \equiv D(v \circ p)\right|_{\Gamma} \text { or }\left(D_{\Gamma} v\right)_{i j}=\left(\nabla_{\Gamma} v_{i}\right)_{j}
$$

the tangential divergence as $\left.\operatorname{div}_{\Gamma} v \equiv \operatorname{div}(v \circ p)\right|_{\Gamma}$, the Hessian $D_{\Gamma}^{2} f$ of $f \in C^{2}(\Gamma)$ as $D_{\Gamma}^{2} f=D_{\Gamma}\left(\nabla_{\Gamma} f\right)$, the Laplace-Beltrami operator of $f \in C^{2}(\Gamma)$ as $\Delta_{\Gamma} f \equiv \operatorname{div}_{\Gamma}\left(\nabla_{\Gamma} f\right)=\left.\Delta(f \circ p)\right|_{\Gamma}$, the tangential linear strain tensor of elasticity as $\varepsilon_{\Gamma}(v) \equiv \frac{1}{2}\left(D_{\Gamma} v+{ }^{*} D_{\Gamma} v\right)=\left.\varepsilon(v \circ p)\right|_{\Gamma}$, and the tangential vectorial divergence of a second-order tensor $A$ as $\left.\operatorname{div}_{\Gamma} A \equiv \operatorname{div}(A \circ p)\right|_{\Gamma}=\operatorname{div}_{\Gamma} A_{i}$. Using the definitions given in section (2) one can derive Green's formula in the tangential calculus [6]:

$$
\int_{\Gamma} f \operatorname{div}_{\Gamma} v d \Gamma+\int_{\Gamma}\left\langle\nabla_{\Gamma} f, v\right\rangle d \Gamma=\int_{\Upsilon}\langle f v, \nu\rangle d \Upsilon+2 \int_{\Gamma} f H\langle v, \nabla b\rangle d \Gamma
$$

where $\nu$ is the outward unit normal to the curve $\Upsilon$. Finally, from $[6,8]$ we have that

$$
\left\langle\nabla_{\Gamma} w, \nabla b\right\rangle=0, \quad D_{\Gamma} v \nabla b=0
$$

by definition for any scalar $w$ and vector $v$. In addition, if we consider a purely tangent vector $v=v_{\Gamma}$, i.e. $\left\langle v_{\Gamma}, \nabla b\right\rangle=0$, we can take the tangential gradient of both sides of this expression and derive

$$
D^{2} b v_{\Gamma}+{ }^{*} D_{\Gamma} v_{\Gamma} \nabla b=0
$$

In this case the first term of equation (4) is also zero. We shall note $|w|_{s, \Gamma} \equiv|w|_{H^{s}(\Gamma)}$ and $(u, v)_{\Gamma} \equiv \int_{\Gamma} u v d \Gamma$ where $\langle\cdot, \cdot\rangle$ denotes the scalar product of two vectors. Throughout this paper the conventions of [9] concerning tensors are used.

Hypothesis 2.1 The following assumptions are imposed on the shell $S_{h}$ with midsurface $\Gamma$.

(i) The shell is assumed to be made of an isotropic and homogeneous material, so that the Lamé coefficients $\lambda>0$ and $\mu>0$ are constant.

(ii) The thickness $h$ of the shell is small enough to accommodate the curvatures $H$ and $K$, i.e. the product of the thickness by the curvatures is small as compared to 1. As a consequence we shall drop terms of order equal or greater than 2 in the series expansions with respect to the radial variable. This also implies that $j(z)=$ $\operatorname{det}\left(D T_{z}\right)=\operatorname{det}\left(I-z D^{2} b\right)=1$. 
(iii) (Kirchhoff Hypothesis) Let $T$ be a transformation of the shell $S_{h}$, and let $\mathbf{e}=\left(e_{\Gamma}, w\right)$ be the corresponding transformation of the midsurface. In the classical thin plate theory named after Kirchhoff, the displacement vectors $T$ and $e \circ p$ are related by the hypothesis that the filaments of the plate initially perpendicular to the middle surface remain straight and perpendicular to the deformed surface, and undergo neither contraction nor extension. We may generalize this hypothesis to the case of a shell using the intrinsic geometry, yielding $T=\mathbf{e} \circ p-b\left({ }^{*} D_{\Gamma_{0}} \mathbf{e} \nabla b\right) \circ p$

(iv) We will assume the boundary $\Upsilon$ consists of two open connected regions $\Upsilon_{0}$ and $\Upsilon_{1}$, with $\Upsilon=\overline{\Upsilon_{0} \cup \Upsilon_{1}}$ and $\emptyset=\Upsilon_{0} \cap \Upsilon_{1}$. We will clamp the shell on $\Upsilon_{0}$, and allow $\Upsilon_{1}$ to be free. $\Upsilon_{1}$ may be empty, in which case we recover the result of [5].

We denote by e the transformation of the shell mid-surface and by $e_{\Gamma}$ and $e_{n}$ the tangential and normal components of $\mathbf{e}$ in local coordinates. We define $w$ to be the magnitude of the normal displacement.

$$
w=\langle\mathbf{e}, \nabla b\rangle, \quad e_{n}=w \nabla b, \quad e_{\Gamma}=\mathbf{e}-e_{n}
$$

Here we list the following definitions and properties derived in [5]:

Lemma 1 The following strain-displacement relation holds for a shell modeled in the intrinsic geometry under Hypothesis 2.1 (i)-(iii).

$$
\begin{aligned}
\varepsilon(T)= & \left(\varepsilon_{\Gamma}\left(e_{\Gamma}\right)+w D^{2} b+V_{\Gamma} e_{\Gamma}\right) \circ p \\
& \quad-b\left(-\varepsilon_{\Gamma}\left(D^{2} b e_{\Gamma}\right)+C_{\Gamma} e_{\Gamma}+S_{\Gamma} w+G_{\Gamma} w+w\left(D^{2} b\right)^{2}\right) \circ p
\end{aligned}
$$

where $\varepsilon_{\Gamma}$ is the tangential linear strain tensor of elasticity and $C_{\Gamma}, V_{\Gamma}$, $G_{\Gamma}$, and $S_{\Gamma}$ are defined by

$$
\begin{aligned}
C_{\Gamma} u & =\frac{1}{2}\left(D^{2} b^{*} D_{\Gamma} u+D_{\Gamma} u D^{2} b\right) \\
V_{\Gamma} u & =\frac{1}{2}\left(\left(D^{2} b u\right) \otimes \nabla b+\nabla b \otimes\left(D^{2} b u\right)\right) \\
G_{\Gamma} w & =\frac{1}{2}\left(\left(\nabla b \otimes \nabla_{\Gamma} w\right) D^{2} b+D^{2} b\left(\nabla_{\Gamma} w \otimes \nabla b\right)\right) \\
S_{\Gamma} w & =\frac{1}{2}\left(D_{\Gamma}^{2} w+{ }^{*} D_{\Gamma}^{2} w\right)
\end{aligned}
$$

$C_{\Gamma}$ and $V_{\Gamma}$ are 1st-order and 0-order operators, respectively, that in practice operate on a tangential vector $u . G_{\Gamma}$ is a first-order operator, and $S_{\Gamma}$ is the symmetrization of the Hessian matrix of a scalar function $w$ (the Hessian matrix is not symmetric in the tangential calculus [6]). 


\section{Weak form of the model}

The elastic energy $\mathcal{E}_{p}$ and kinetic energy $\mathcal{E}_{k}$ of the shell under Hypothesis 2.1 were derived in [5]. The coefficient of rotational inertia is denoted $\gamma=\frac{h^{2}}{12}$.

$$
V=\left\{\mathbf{e} \in\left[H^{1}(\Gamma)\right]^{2} \times H^{2}(\Gamma) \mid e_{\Gamma}=w=\frac{\partial}{\partial \nu} w=0 \text { on } \Upsilon_{0}\right\}
$$

We wish to define the weak form of the model in terms of two symmetric bilinear forms, $\mathfrak{m}(\mathbf{e}, \hat{\mathbf{e}})$ and $\mathfrak{a}(\mathbf{e}, \hat{\mathbf{e}})$.

Theorem 2 (Weak form of the model) The displacement of the midsurface of the shell e satisfies the following equality:

$$
\int_{0}^{\tau}\left[-\mathfrak{m}\left(\partial_{t} \mathbf{e}, \partial_{t} \hat{\mathbf{e}}\right)+\mathfrak{a}(\mathbf{e}, \hat{\mathbf{e}})\right] d t=0
$$

for all test functions $\hat{\mathbf{e}} \in V$, with $\mathfrak{m}(\mathbf{e}, \hat{\mathbf{e}})$ and $\mathfrak{a}(\mathbf{e}, \hat{\mathbf{e}})$ given by

$$
\begin{gathered}
-\mathfrak{m}(\mathbf{e}, \hat{\mathbf{e}})=- \\
-\gamma\left[2\left(e_{\Gamma}, \hat{e_{\Gamma}}\right)_{\Gamma}+2 \gamma\left(\left(D^{2} b\right)_{\Gamma},\left(D^{2} b\right) \hat{e_{\Gamma}}\right)_{\Gamma}-\gamma\left(\nabla_{\Gamma} w,\left(D^{2} b\right) \hat{e_{\Gamma}}\right)_{\Gamma}\right. \\
\left.\mathfrak{a}(\mathbf{e}, \hat{\mathbf{e}})=2 \lambda \gamma\left(\Delta_{\Gamma} w, \Delta_{\Gamma} \hat{w}\right)_{\Gamma}+4 \mu \gamma\left(w, \operatorname{tr}\left(S_{\Gamma} \hat{w}\left(D^{2} b\right)^{2}\right)\right)_{\Gamma}+2(w, \hat{w})_{\Gamma}+2 \gamma\left(\nabla_{\Gamma} w, \nabla_{\Gamma} \hat{w}\right)_{\Gamma}\right] \\
+4 \mu \gamma\left(\operatorname{tr}\left(S_{\Gamma} w\left(D^{2} b\right)^{2}\right), \hat{w}\right)_{\Gamma}+4 \lambda\left(H w, \operatorname{div}_{\Gamma} \hat{e_{\Gamma}}\right)_{\Gamma} \\
+4 \lambda\left(\operatorname{div}_{\Gamma} e_{\Gamma}, H \hat{w}\right)_{\Gamma}+4 \mu\left(w, \operatorname{tr}\left(\varepsilon_{\Gamma}\left(\hat{e_{\Gamma}}\right) D^{2} b\right)\right)_{\Gamma} \\
-4 \mu \gamma\left(\operatorname{tr}\left(D^{3} b e_{\Gamma}\left(D^{2} b\right)^{2}\right), \hat{w}\right)_{\Gamma}+4 \mu\left(\operatorname{tr}\left(\varepsilon_{\Gamma}\left(e_{\Gamma}\right) D^{2} b\right), \hat{w}\right)_{\Gamma} \\
+2\left(\sqrt{k_{\gamma}} w, \sqrt{k_{\gamma}} \hat{w}\right)_{\Gamma}+2 \lambda \gamma\left(\operatorname{div}_{\Gamma}\left(D^{2} b e_{\Gamma}\right), \operatorname{div}_{\Gamma}\left(D^{2} b \hat{e_{\Gamma}}\right)\right)_{\Gamma} \\
-2 \mu\left(D^{2} b e_{\Gamma}, D^{2} b \hat{e_{\Gamma}}\right)_{\Gamma}-2 \lambda \gamma\left(\operatorname{div}_{\Gamma}\left(D^{2} b e_{\Gamma}\right), \operatorname{tr}\left(C_{\Gamma} \hat{e_{\Gamma}}\right)\right)_{\Gamma} \\
-2 \lambda \gamma\left(\operatorname{tr}\left(C_{\Gamma} e_{\Gamma}\right), \operatorname{div}_{\Gamma}\left(D^{2} b \hat{e_{\Gamma}}\right)\right)_{\Gamma}+2 \lambda \gamma\left(\operatorname{tr}\left(C_{\Gamma} e_{\Gamma}\right), \operatorname{tr}\left(C_{\Gamma} \hat{e_{\Gamma}}\right)\right)_{\Gamma} \\
-2 \lambda \gamma\left(\operatorname{tr}\left(D^{3} b e_{\Gamma}\right), \Delta_{\Gamma} \hat{w}\right)_{\Gamma}-2 \lambda \gamma\left(\Delta_{\Gamma} w, \operatorname{tr}\left(D^{3} b \hat{e_{\Gamma}}\right)\right)_{\Gamma} \\
-2 \lambda \gamma\left(\left(4 H^{2}-2 K\right) w, \operatorname{tr}\left(D^{3} b \hat{e_{\Gamma}}\right)\right)_{\Gamma}-2 \lambda \gamma\left(\operatorname{tr}\left(D^{3} b e_{\Gamma}\right),\left(4 H^{2}-2 K\right) \hat{w}\right)_{\Gamma} \\
+2 \lambda\left(\operatorname{div}_{\Gamma} e_{\Gamma}, \operatorname{div}_{\Gamma} \hat{e_{\Gamma}}\right)_{\Gamma}+2 \lambda \gamma\left(\left(4 H^{2}-2 K\right) w, \Delta_{\Gamma} \hat{w}\right)_{\Gamma} \\
+2 \lambda \gamma\left(\Delta_{\Gamma} w,\left(4 H^{2}-2 K\right) \hat{w}\right)_{\Gamma}-4 \mu \gamma\left(w, \operatorname{tr}\left(D^{3} b \hat{e_{\Gamma}}\left(D^{2} b\right)^{2}\right)\right)_{\Gamma} \\
-4 \mu \gamma \int_{\Gamma} \operatorname{tr}\left(\varepsilon_{\Gamma}\left(D^{2} b e_{\Gamma}\right) S_{\Gamma} \hat{w}\right)+4 \mu \int_{\Gamma} \operatorname{tr}\left(\varepsilon_{\Gamma}\left(e_{\Gamma}\right) \varepsilon_{\Gamma}\left(\hat{e_{\Gamma}}\right)\right) \\
+4 \mu \gamma \int_{\Gamma} \operatorname{tr}\left(\varepsilon_{\Gamma}\left(D^{2} b e_{\Gamma}\right) \varepsilon_{\Gamma}\left(D^{2} b \hat{e_{\Gamma}}\right)\right)+4 \mu \gamma \int_{\Gamma} \operatorname{tr}\left(C_{\Gamma} e_{\Gamma} C_{\Gamma} \hat{e_{\Gamma}}\right) \\
-4 \mu \gamma \int_{\Gamma} \operatorname{tr}\left(C_{\Gamma} e_{\Gamma} \varepsilon_{\Gamma}\left(D^{2} b \hat{e_{\Gamma}}\right)\right)-4 \mu \gamma \int_{\Gamma} \operatorname{tr}\left(\varepsilon_{\Gamma}\left(D^{2} b e_{\Gamma}\right) C_{\Gamma} \hat{e_{\Gamma}}\right) \\
+
\end{gathered}
$$




$$
\begin{gathered}
+4 \mu \gamma \int_{\Gamma} \operatorname{tr}\left(C_{\Gamma} e_{\Gamma} S_{\Gamma} \hat{w}\right)+4 \mu \gamma \int_{\Gamma} \operatorname{tr}\left(S_{\Gamma} w C_{\Gamma} \hat{e_{\Gamma}}\right) \\
+4 \mu \gamma \int_{\Gamma} \operatorname{tr}\left(\left(S_{\Gamma} w+G_{\Gamma} w\right)\left(S_{\Gamma} \hat{w}+G_{\Gamma} \hat{w}\right)\right)-4 \mu \gamma \int_{\Gamma} \operatorname{tr}\left(S_{\Gamma} w \varepsilon_{\Gamma}\left(D^{2} b \hat{e_{\Gamma}}\right)\right)
\end{gathered}
$$

Proof. Let us consider a final time $\tau$. Among all kinematically admissible displacements, the actual motion of the shell will make stationary the Lagrangian $\mathcal{L}(\mathbf{e})=\int_{0}^{\tau} \mathcal{E}_{k}(\mathbf{e})-\mathcal{E}_{p}(\mathbf{e})$. We consider the Gâteaux-derivative in a direction $\hat{\mathbf{e}}$,

$$
\forall \hat{\mathbf{e}},\left.\frac{\partial \mathcal{L}(\mathbf{e}+\theta \hat{\mathbf{e}})}{\partial \theta}\right|_{\theta=0}=0
$$

with $\hat{\mathbf{e}}(0)=\partial_{t} \hat{\mathbf{e}}(0)=\hat{\mathbf{e}}(\tau)=\partial_{t} \hat{\mathbf{e}}(\tau)=0$. Simplifying and using and using various properties of the operators defined in (9) gives $\mathfrak{a}(\mathbf{e}, \hat{\mathbf{e}})$ in the required form. The constant $k_{\gamma}$ (a positive real number which depends on the curvature, the Lamé coefficients, and the rotational inertia coefficient) is defined by

$$
\begin{aligned}
k_{\gamma}=4 H^{2} \lambda+\left(8 H^{2}-4 K\right) \mu+\gamma( & \left(4 H^{2}-2 K\right)^{2} \lambda \\
& \left.+2\left(16 H^{2}-16 H^{2} K+2 K^{2}\right) \mu\right)>0
\end{aligned}
$$

Proposition 3 (Ellipticity of the strain operator) The bilinear form $\mathfrak{a}(\mathbf{e}, \mathbf{e})$ defined in (13) is elliptic on $V$ where the space $V$ is defined by $(10)$.

Proof. It is shown in [5] that the strain tensor presented in (8) is identical to the Koiter shell model with 'modified' change of curvature tensor. Thus, as long as the shell is clamped on part of the boundary, the proof of Proposition 3 follows directly from the work of Bernadou and Ciarlet[3] (see also [4], and [1] pp. 23-30).

Proposition 4 (Existence, uniqueness of weak solutions) Let $\Gamma$ be a bounded open set with boundary $\Upsilon$ as previously described. Then there exists a unique solution $\mathbf{e} \in C([0, \infty) ; V)$ to the problem $(11)$, with the space $V$ given by (10).

Proof. The proof follows immediately from Proposition 3 and the LaxMilgram theorem. 


\section{Strong form of the model}

Here we wish to integrate the symmetric bilinear forms given in Theorem 2 in order to derive a strong form of the model and the associated boundary conditions on the free part of the boundary $\Upsilon_{1}$. Essentially, we wish to define two operators, $\mathcal{M}_{\gamma}$ and $\mathcal{A}$, so that $\mathcal{M}_{\gamma}$ corresponds to the kinetic energy of the model and $\mathcal{A}$ to the potential energy. Integration will give the strong form of $\mathcal{M}_{\gamma}, \mathcal{A}$. In [5] this calculation is done assuming a test function $\hat{\mathbf{e}}$ which is identically zero on the boundary $\Upsilon$, as a shell with fully clamped boundary was considered. By contrast here we consider a shell which is clamped on only part of the boundary, $\Upsilon_{0}$, and is free on $\Upsilon_{1}$.

Theorem 5 (Strong form of the model) Define the following operator $\mathcal{C}$ acting on a matrix $A$ :

$$
\mathcal{C}(A)=\lambda \operatorname{tr}(A) I+2 \mu A
$$

the expression $\tilde{\chi}$

$$
\tilde{\chi}=C_{\Gamma} e_{\Gamma}-\varepsilon_{\Gamma}\left(D^{2} b e_{\Gamma}\right)
$$

and the parameter $\beta=(\lambda+2 \mu)^{-1}$. Then, $\mathbf{e} \in C([0, \infty) ; V)$ satisfies the following system of shell equations which holds on $\Gamma \times(0, \infty)$ :

$$
\begin{gathered}
\partial_{t t} w-\gamma \Delta_{\Gamma} \partial_{t t} w+\Delta_{\Gamma}^{2} w+\frac{\gamma}{2} \operatorname{div}_{\Gamma}\left(D^{2} b \partial_{t t} e_{\Gamma}\right)+P_{1}\left(e_{\Gamma}\right)+Q_{1}(w)=0 \\
\left(I+\gamma\left(D^{2} b\right)^{2}\right) \partial_{t t} e_{\Gamma}-\beta\left[\gamma^{-1} \operatorname{div}_{\Gamma} \mathcal{C}\left(\varepsilon_{\Gamma}\left(e_{\Gamma}\right)\right)+D^{2} b \operatorname{div}_{\Gamma} \mathcal{C}(\tilde{\chi})\right. \\
\left.-\operatorname{div}_{\Gamma}\left(D^{2} b \mathcal{C}(\tilde{\chi})\right)\right]-\frac{\gamma}{2} D^{2} b \nabla_{\Gamma} \partial_{t t} w+P_{2}(w)+Q_{2}\left(e_{\Gamma}\right)=0
\end{gathered}
$$

where $P_{1}$ denotes coupling terms and $Q_{1}$ denotes lower order terms in the plate equation; and $P_{2}, Q_{2}$ in the wave equation:

$$
\begin{aligned}
P_{1}\left(e_{\Gamma}\right)=\beta[2 \lambda & H \gamma^{-1} \operatorname{div}_{\Gamma} e_{\Gamma}+2 \mu \gamma^{-1} \operatorname{tr}\left(D^{2} b \varepsilon_{\Gamma}\left(e_{\Gamma}\right)\right) \\
& +2 \mu \operatorname{div}_{\Gamma} \operatorname{div}_{\Gamma}(\tilde{\chi})+4 \mu H \operatorname{tr}\left(D^{3} b e_{\Gamma} D^{2} b\right) \\
& -\lambda \Delta_{\Gamma}\left\langle 2 \nabla_{\Gamma} H, e_{\Gamma}\right\rangle-\lambda\left(4 H^{2}-2 K\right)\left\langle 2 \nabla_{\Gamma} H, e_{\Gamma}\right\rangle(17 \mathrm{a}) \\
& -2 \mu\left\langle\left(D^{2} b\right)^{2} . . D^{3} b, e_{\Gamma}\right\rangle \\
Q_{1}(w)=\beta\left[k_{\gamma} w\right. & +4 \mu \operatorname{div}_{\Gamma}\left(\left(D^{2} b\right)^{2} \nabla_{\Gamma} w\right)+\lambda \Delta_{\Gamma}\left(\left(4 H^{2}-2 K\right) w\right) \\
& +2 \mu \operatorname{div}_{\Gamma} \operatorname{div}_{\Gamma}\left(\left(D^{2} b\right)^{2} w\right)+\lambda\left(4 H^{2}-2 K\right) \Delta_{\Gamma} w \\
& \left.+2 \mu \operatorname{div}_{\Gamma}\left(K \nabla_{\Gamma} w\right)\right]+2 \mu \operatorname{tr}\left(S_{\Gamma} w\left(D^{2} b\right)^{2}\right) \\
& +4 \mu H \operatorname{tr}\left(\left(D^{2} b\right)^{3} w\right)
\end{aligned}
$$




$$
\begin{aligned}
P_{2}(w)= & \beta\left[-2 \lambda \gamma^{-1} \nabla_{\Gamma}(H w)+2 \mu \gamma^{-1} \operatorname{div}_{\Gamma}\left(w D^{2} b\right)\right. \\
& \left.+\lambda 2 \nabla_{\Gamma} H\left(\Delta_{\Gamma} w-\left(4 H^{2}-2 K\right) w\right)-2 \mu\left(D^{2} b\right)^{2} . . D^{3} b w\right] \\
& -2 \mu \operatorname{div}_{\Gamma}\left(D^{2} b S_{\Gamma} w\right)+2 \mu D^{2} b \operatorname{div}_{\Gamma}\left(S_{\Gamma} w\right) \\
Q_{2}\left(e_{\Gamma}\right)= & -2 \beta \mu \gamma^{-1}\left(K e_{\Gamma}+2\left(D^{2} b\right)^{2} e_{\Gamma}\right)
\end{aligned}
$$

the following free boundary conditions on $\Upsilon_{1} \times(0, \infty)$ :

$$
\begin{aligned}
& \mathcal{C}\left(w D^{2} b+\varepsilon_{\Gamma}\left(e_{\Gamma}\right)\right) \cdot \nu=0 \\
& \left\langle\left(\lambda \beta \nabla_{\Gamma} \operatorname{tr}\left(D^{3} b e_{\Gamma}\right)+4 \mu \beta\left(D^{2} b\right)^{2} \nabla_{\Gamma} w+2 \mu \beta K \nabla_{\Gamma} w-\nabla_{\Gamma}\left(\Delta_{\Gamma} w\right), \nu\right\rangle\right. \\
& \quad+\left\langle\gamma\left(D^{2} b \partial_{t t} e_{\Gamma}-2 \nabla_{\Gamma} \partial_{t t} w\right)+2 \mu \beta B_{\Gamma}^{3}\left(C_{\Gamma} e_{\Gamma}-\varepsilon_{\Gamma}\left(D^{2} b e_{\Gamma}\right)\right.\right. \\
& \left.\left.\quad+\left(D^{2} b\right)^{2} w\right), \nu\right\rangle-\beta \lambda\left\langle\nabla_{\Gamma}\left(\left(4 H^{2}-2 K\right) w\right), \nu\right\rangle+2 \mu B_{\Gamma}^{2} w=0 \\
& \lambda \beta \operatorname{tr}\left(D^{3} b e_{\Gamma}\right)+\lambda \beta\left(4 H^{2}-2 K\right) w-\Delta_{\Gamma} w \\
& \quad+2 \mu \beta\left\langle\left(C_{\Gamma} e_{\Gamma}-\varepsilon_{\Gamma}\left(D^{2} b e_{\Gamma}\right)+\left(D^{2} b\right)^{2}\right) \nu, \nu\right\rangle+2 \mu \beta B_{\Gamma}^{1} w=0
\end{aligned}
$$

and clamped boundary conditions on $\Upsilon_{0} \times(0, \infty)$ :

$$
w=\frac{\partial}{\partial \nu} w=e_{\Gamma}=0
$$

Proof. We search for the explicit representations of the following Green'stype formulas:

$$
\begin{gathered}
\int_{0}^{\tau}-\mathfrak{m}\left(\partial_{t} \mathbf{e}, \partial_{t} \hat{\mathbf{e}}\right) d t= \\
\int_{0}^{\tau} \int_{\Gamma}\left\langle\mathcal{M}_{\gamma} \partial_{t t} \mathbf{e}, \hat{\mathbf{e}}\right\rangle d t d \Gamma-\int_{0}^{\tau} \int_{\Upsilon_{1}} \mathfrak{B}_{\mathcal{M}}\left(\partial_{t t} \mathbf{e}, \hat{\mathbf{e}}\right) d t d \Upsilon_{1} \\
(\mathcal{A} \mathbf{e}, \hat{\mathbf{e}})_{\Gamma}=\int_{\Gamma}\langle\mathcal{A} \mathbf{e}, \hat{\mathbf{e}}\rangle d \Gamma=\int_{\Upsilon_{1}} \mathfrak{B}_{\mathcal{A}}(\mathbf{e}, \hat{\mathbf{e}}) d \Upsilon_{1}+\mathfrak{a}(\mathbf{e}, \hat{\mathbf{e}})
\end{gathered}
$$

where $\mathfrak{B}(\mathbf{e}, \hat{\mathbf{e}})$ are expressions containing boundary terms only. We first note that the regularity of the weak solutions is high enough to permit the necessary integration by parts to derive the strong form.

Proposition 6 Let $\Gamma$ with boundary $\Upsilon$ be as previously described and assume e $\in C((0, \infty) ; V)$ such that

$$
\int_{0}^{\tau}\left[-\mathfrak{m}\left(\partial_{t} \mathbf{e}, \partial_{t} \hat{\mathbf{e}}\right)+\mathfrak{a}(\mathbf{e}, \hat{\mathbf{e}})\right] d t=0 \quad \forall \hat{\mathbf{e}} \in V
$$


with the space $V$ defined in (10). Then $\mathbf{e} \in C\left((0, \infty) ; V_{1}\right)$ where

$$
V_{1}=\left\{\mathbf{e} \in\left[H^{2}(\Gamma)\right]^{2} \times H^{4}(\Gamma) \mid e_{\Gamma}=w=\frac{\partial}{\partial \nu} w=0 \text { on } \Upsilon_{0}\right\}
$$

and the restriction of $\mathbf{e}$ to the boundary $\mathbf{e} \mid \Upsilon \in\left[H^{1 / 2}(\Upsilon)\right]^{2} \times H^{3 / 2}(\Upsilon)$.

Proof. Choose $\hat{\mathbf{e}} \in[\mathcal{D}(\Gamma)]^{3}$. Then the integrals over $\Upsilon$ in (19) and (20) vanish so that we have

$$
\int_{0}^{\tau} \int_{\Gamma}\left\langle\mathcal{M}_{\gamma} \partial_{t t} \mathbf{e}+\mathcal{A} \mathbf{e}, \hat{\mathbf{e}}\right\rangle d \Gamma d t=0, \quad \forall \hat{\mathbf{e}} \in[\mathcal{D}(\Gamma)]^{3}
$$

Thus $\mathcal{M}_{\gamma} \partial_{t t} \mathbf{e}+\mathcal{A} \mathbf{e}=0$ in the sense of distributions. This equality also holds in $\left[L_{2}(\Gamma)\right]^{3}$, since $[\mathcal{D}(\Gamma)]^{3}$ is dense in $\left[L_{2}(\Gamma)\right]^{3}$ and the $\Gamma$ integrals are continuous with respect to $\hat{\mathbf{e}}$ in the $L_{2}(\Gamma)$ topology. Next, we consider an arbitrary ê and integrate (21), giving

$$
\int_{0}^{\tau} \int_{\Gamma}\left\langle\mathcal{M}_{\gamma} \partial_{t t} \mathbf{e}+\mathcal{A} \mathbf{e}, \hat{\mathbf{e}}\right\rangle d \Gamma d t-\int_{0}^{\tau} \int_{\Upsilon} \mathfrak{B}_{\mathcal{M}}\left(\partial_{t t} \mathbf{e}, \hat{\mathbf{e}}\right)+\mathfrak{B}_{\mathcal{A}}(\mathbf{e}, \hat{\mathbf{e}}) d \Upsilon d t=0
$$

By the previous step this gives immediately that

$$
\int_{0}^{\tau} \int_{\Upsilon} \mathfrak{B}_{\mathcal{M}}\left(\partial_{t t} \mathbf{e}, \hat{\mathbf{e}}\right)+\mathfrak{B}_{\mathcal{A}}(\mathbf{e}, \hat{\mathbf{e}}) d \Upsilon_{1} d t=0
$$

for any $\hat{\mathbf{e}} \in\left[H^{1}(\Gamma)\right]^{2} \times H^{2}(\Gamma)$. However, for any $\left.\hat{\mathbf{e}}\right|_{\Upsilon} \in\left[H^{1 / 2}(\Upsilon)\right]^{2} \times$ $H^{3 / 2}(\Upsilon)$, by surjectivity of the trace map there exists an extension $\tilde{\hat{\mathbf{e}}} \in\left[H^{1}(\Gamma)\right]^{2} \times H^{2}(\Gamma)$ such that $\tilde{\hat{\mathbf{e}}}|\Upsilon=\hat{\mathbf{e}}| \Upsilon$. This gives that $(25)$ holds true for all $\hat{\mathbf{e}} \mid \Upsilon \in\left[H^{1 / 2}(\Upsilon)\right]^{2} \times H^{3 / 2}(\Upsilon)$, and thus again by density we have that $\mathfrak{B}_{\mathcal{M}} \partial_{t t} \mathbf{e}+\mathfrak{B}_{\mathcal{A}} \mathbf{e}=0$ in $\left[L^{2}(\Upsilon)\right]^{3}$.

With respect to the kinetic energy, integration by parts in time followed by an application of the Green's formula (4) gives that

$$
\begin{gathered}
\left(\mathcal{M}_{\gamma} \partial_{t t} \mathbf{e}, \hat{\mathbf{e}}\right)_{\Gamma}=\rho\left[\left(2 \partial_{t t} e_{\Gamma}+2 \gamma\left(D^{2} b\right)^{2} \partial_{t t} e_{\Gamma}, \hat{e_{\Gamma}}\right)_{\Gamma}\right. \\
-\gamma\left(\left(D^{2} b\right) \nabla_{\Gamma} \partial_{t t} w, \hat{e_{\Gamma}}\right)_{\Gamma}+\gamma\left(\operatorname{div}_{\Gamma}\left(\left(D^{2} b\right) \partial_{t t} e_{\Gamma}\right), \hat{w}\right)_{\Gamma} \\
\left.+\left(2 \partial_{t t} w-2 \gamma \Delta_{\Gamma} \partial_{t t} w, \hat{w}\right)_{\Gamma}\right] \\
\int_{\Upsilon_{1}} \mathfrak{B}_{\mathcal{M}}\left(\partial_{t t} \mathbf{e}, \hat{\mathbf{e}}\right) d \Upsilon_{1}=-\rho \gamma \int_{\Upsilon_{1}}\left(\left(D^{2} b\right) \partial_{t t} e_{\Gamma} \cdot \nu-2 \nabla_{\Gamma} \partial_{t t} w \cdot \nu\right) \hat{w} d \Upsilon_{1}
\end{gathered}
$$

We note that terms involving the principal curvature $H$ coming from (4) are zero since both $\nabla_{\Gamma} \hat{w}$ and $D^{2} b \hat{e_{\Gamma}}$ are tangential vectors. Now we 
need to integrate each of the thirty-two terms of (13) to derive a strong representation of the operator $\mathcal{A}$. Space considerations do not allow us to show all of these calculations here, thus we note only the procedure followed, especially that with the highest-order terms. The first term of (13) will yield the required tangential biharmonic operator $\Delta_{\Gamma}^{2}$ in the strong form. However, the second term of this equation is also a fourthorder operator, and analogously with the plate case we desire to combine the two terms $\Delta_{\Gamma} w \Delta_{\Gamma} \hat{w}$ and $\operatorname{tr}\left(S_{\Gamma} w S_{\Gamma} \hat{w}\right)$. The problem here lies in the fact that the matrix $D_{\Gamma}^{2} w$ is not symmetric and is not equivalent to the restriction of the Hessian matrix of the canonical extension $w \circ p$. In fact the two terms differ by a first-derivative correction, as is seen in the following identity [6]: $D_{\Gamma}^{2} w-\left(D^{2} b \nabla_{\Gamma} w\right) \otimes \nabla b=\left.D^{2}(w \circ p)\right|_{\Gamma}$. We use this identity and the definitions of the operators $S_{\Gamma}$ and $G_{\Gamma}$ to write that

$$
\begin{gathered}
2 \lambda \gamma\left(\Delta_{\Gamma} w, \Delta_{\Gamma} \hat{w}\right)_{\Gamma}+4 \mu \gamma \int_{\Gamma} \operatorname{tr}\left(\left(S_{\Gamma} w+G_{\Gamma} w\right)\left(S_{\Gamma} \hat{w}+G_{\Gamma} \hat{w}\right)\right) d \Gamma= \\
2 \gamma(\lambda+2 \mu)\left(\Delta_{\Gamma} w, \Delta_{\Gamma} \hat{w}\right)_{\Gamma}-8 \mu \gamma\left(D^{2} b \nabla_{\Gamma} w, D^{2} b \nabla_{\Gamma} \hat{w}\right)_{\Gamma}+4 \mu \gamma \int_{\Gamma} \text { a.t. } d \Gamma
\end{gathered}
$$

where a.t. stands for additional terms. Analogous to the plate case we are able to convert the additional term in this equation to an integral over the boundary $\Upsilon$ by showing that it is the divergence of an appropriate vector. In fact the last integral is equivalent to the following: where

$$
4 \mu \gamma \int_{\Upsilon}\left[B_{\Gamma}^{1}\left(\left.D^{2}(w \circ p)\right|_{\Gamma}\right) \nabla_{\Gamma} \hat{w} \cdot \nu-\hat{w} B_{\Gamma}^{2}\left(\left.D^{2}(w \circ p)\right|_{\Gamma}\right)\right] d \Upsilon
$$

$$
\begin{aligned}
& B_{\Gamma}^{1} A=-(\tau \otimes \tau) . . A \\
& B_{\Gamma}^{2} A=\left\langle\nabla_{\Gamma}((\tau \otimes \nu) . . A), \tau\right\rangle
\end{aligned}
$$

with $\nu$ and $\tau$ being the normal and tangent vectors to the boundary $\Upsilon$. Denoting $\nu=\left(\nu_{1}, \nu_{2}\right)$ and $\tau=\left(-\nu_{2}, \nu_{1}\right)$ and expanding equations (29) shows that the $B_{\Gamma}^{1}\left(\left.D^{2}(w \circ p)\right|_{\Gamma}\right)$ and $B_{\Gamma}^{2}\left(\left.D^{2}(w \circ p)\right|_{\Gamma}\right)$ in (28) are exactly the restriction to $\Gamma$ of the standard $B_{1}, B_{2}$ operators (which appear in the modeling of Kirchhoff plates) operating on the canonical extension $w \circ p$ (see, e.g., [14], [15]). Integration of this term and the others follow through explicit use of (4), (6) and (5). For readability we define also

$$
B_{\Gamma}^{3} A=B_{\Gamma}^{2} A+\left\langle\operatorname{div}_{\Gamma} A, \nu\right\rangle
$$

and the following operator $\mathcal{C}$ acting on a matrix $A: \mathcal{C}(A)=\lambda \operatorname{tr}(A) I+$ $2 \mu A$. Finally, we make a change of time variable $t \rightarrow t \sqrt{\frac{\gamma(\lambda+2 \mu)}{\rho}}$ in both equations and divide both equations by the term $2 \gamma(\lambda+2 \mu)$. Defining $\beta=(\lambda+2 \mu)^{-1}$ gives the final form of the equations. 
Theorem 7 (Existence and uniqueness of strong solutions) Let $\Gamma$ be a bounded open set with boundary $\Upsilon$ as previously described. Then there exists a unique solution $\mathbf{e} \in C((0, \infty) ; V)$ to the system of equations presented in Theorem 4, with the space $V$ given by (10).

Proof. The proof follows immediately from the Lumer-Phillips theorem once Proposition 3 is established.

\section{References}

[1] M. Bernadou. Finite Element Methods for Thin Shell Problems. Wiley and Sons, 1996.

[2] M. Bernadou and J. M. Boisserie. The Finite Element Method for Thin Shell Problems. Birkhauser, Boston, 1982.

[3] M. Bernadou and P. G. Ciarlet. Sur l'ellipticité du modèle linéare de coques de W. T. Koiter. In Computing Methods in Applied Sciences and Enginnering, Lecture Notes in Economics and Mathematical Systems, vol. 34, pp. 89-136. Springer-Verlag, 1976.

[4] M. Bernadou, P. G. Ciarlet, and B. Miara. Existence theorems for twodimensional linear shell theories. J. Elasticity, 34:111-138, 1994.

[5] J. Cagnol, I. Lasiecka, C. Lebiedzik, and J.-P. Zolésio. Uniform stability in structural acoustic model with flexible curved walls. J.Diff.Eqns., 186(1), 88$121,2002$.

[6] M. Delfour and J. P. Zolésio. Intrinsic differential geometry and theory of thin shells. to appear, 2002.

[7] M. C. Delfour and J.-P. Zolésio. A boundary differential equation for thin shells. Journal of Differential Equations, 119(2):426-449, 1995.

[8] M. C. Delfour and J.-P. Zolésio. Differential equations for linear shells: comparison between intrinsic and classical models. In Advances in mathematical sciences: CRM's 25 years CRM Proc. Lecture Notes, vol. 11, pp. 481-491. AMS 1997.

[9] P. Germain. Mecanique, vol. 1. Ecole Polytechnique, 1986.

[10] G. Kirchhoff. Vorlesungen uber Mathematische Physik. Mechanik, Leipzig, 1876.

[11] W. T. Koiter. A consistent first approximation in the general theory of thin shells. In Proc. Symp. on Theory of Thin Elastic Shells, pp. 12-33. held in Delft, August 1959.

[12] W. T. Koiter. On the foundation of the linear theory of thin elastic shells. Proc. Kon. Nederl. Akad. Wetensch., B73:169-195, 1970.

[13] J. Lagnese. Boundary Stabilization of Thin Plates. SIAM, Philadelphia, PA, 1989.

[14] J. Lagnese and J.-L. Lions. Modelling analysis and control of thin plates, vol. 6 of Research in Applied Mathematics. Masson, Paris, 1988.

[15] I. Lasiecka and R. Triggiani. Control Theory for Partial Differential Equations, vol. I and II. Encyclopedia of Mathematics and its Applications. Cambridge U. Press, 1999.

[16] A. E. H. Love. The Mathematical Theory of Elasticity. Cambridge Univ. Press, 1934 . 